\title{
Timing of onset of lithium relapse prevention in bipolar disorder: evidence from randomised trials
}

\author{
Matthew J. Taylor
}

\begin{abstract}
Summary
Lithium is widely prescribed, but the timing of key effects remains uncertain. The timing of onset of its relapse prevention effects is clarified by placebo-controlled randomised trials (3 studies, $n=1120$ ). Lithium reduced relapse into any mood episode over the first 2 weeks of treatment (hazard ratio 0.40, 95\% $\mathrm{Cl} 0.16-0.97)$. Fewer manic relapses were evident within the first 4 weeks, however, early effects on depressive relapse were not demonstrated. There is an early onset of lithium relapse prevention effects in bipolar disorder, particularly against manic relapse. Full effects against depressive relapse may develop over a longer period.
\end{abstract}

\section{Declaration of interest}

M.J.T. reports personal fees from Sunovion, Otsuka, Lundbeck, outside the submitted work.

\section{Keywords}

Bipolar affective disorders; mood stabilisers; randomized controlled trial.

\section{Copyright and usage}

(c) The Royal College of Psychiatrists 2018.
Lithium is a key agent in the treatment of bipolar disorder, with well-established actions in relapse prevention. ${ }^{1}$ However, ongoing uncertainty around its underlying mechanisms ${ }^{2}$ complicates efforts to develop improved future treatments. A clear understanding of the timing of clinical effects provides information not just of clinical relevance, but can also clarify fundamental underlying biological mechanisms - for example through constraining potential models of action. Lithium has a wide variety of biological effects, but none of the hypotheses for its mechanism of action have been conclusively confirmed. ${ }^{2}$ The timing of onset of its prophylactic effect was recently highlighted as a key uncertainty. ${ }^{2}$ Lithium must have some rapid central effects, given its effectiveness in the acute treatment of manic episodes over a periods of days or weeks, however, it is often suggested that beneficial preventative effects may take a long time to become apparent. $^{3,4}$

Combining data from multiple clinical trials is an approach that combines the methodological power of experimental intervention with sufficient statistical power to demonstrate potentially subtle early effects. In other areas of psychiatry, such an approach has successfully clarified timing of clinical effects. ${ }^{5}$ Here we sought to investigate the early effects of lithium on relapse rates in bipolar disorder through meta-analysis of survival curve data from placebo-controlled randomised controlled trials.

\section{Method}

Placebo-controlled randomised clinical trials of lithium for relapse prevention in bipolar disorder were identified from an existing systematic review and meta-analysis ${ }^{1}$ (original protocol in the Cochrane Library), search updated to May 2018 (see supplementary material available online at https://doi.org/10.1192/bjp.2018.177). From these, studies were identified that presented survival curve data on relapse with weekly resolution of data. In studies with more than two treatment arms, outcome data from those not randomised to placebo or lithium were not used in this analysis.

Survival curve data were extracted using a plot digitiser (version 3.11, https://automeris.io/WebPlotDigitizer/). Interval hazard ratios (HRs) and their variance were estimated as previously described. ${ }^{6,7}$ HRs for those randomised to lithium versus those randomised to placebo in each trial were calculated for relapse into any mood episode. Pooled estimates of HRs and 95\% CI were obtained by inverse variance meta-analysis with a random-effects model. Heterogeneity was assessed by use of $I^{2}$. Relapses into mania and depression were also assessed separately. Given these pole-specific relapses necessarily involved fewer events, HRs were calculated over longer intervals, providing reduced temporal resolution. Analyses were performed using $R$ (version 3.3.2), with meta package (version 4.7).

\section{Results}

Three placebo-controlled randomised clinical trials of relapse prevention in bipolar disorder were identified for inclusion. ${ }^{8-10}$ The studies had included lithium as an active comparator; two were designed to investigate lamotrigine ${ }^{8,9}$ and one quetiapine, ${ }^{10}$ and are described further elsewhere. ${ }^{1}$ A total of 1120 people with DSM-IV bipolar I disorder had been randomised to either lithium or placebo, of whom $40 \%$ had an index depressive episode and $60 \%$ had an index manic or mixed episode.

\section{Relapse}

For relapse into any mood episode, an early reduction in risk of relapse was seen in those randomised to lithium. Between baseline and week 2 , those randomised to receive lithium were significantly less likely to relapse than those randomised to placebo $(\mathrm{HR}=0.40$, 95\% CI 0.16-0.97). Similarly, a significant reduction was seen between week 2 and week 4 ( $\mathrm{HR}=0.62,95 \%$ CI $0.41-0.93)$ and similar effects seen over following intervals (Fig. 1(a)), although the effect over the final time interval assessed did not reach statistical significance ( $\mathrm{HR}=0.50,95 \% \mathrm{CI} 0.24-1.03)$. Substantial heterogeneity between studies was evident for this final estimate $\left(I^{2}=87 \%\right.$; Fig. 1(b)).

\section{Manic relapse}

For risk of manic relapse, risk was lower in those randomised to receive lithium between baseline and week $4, \mathrm{HR}=0.41$ (95\% CI $0.24-0.73$ ), with a similar effect between week 4 and week 15 , HR 
(a)

Any mood episode

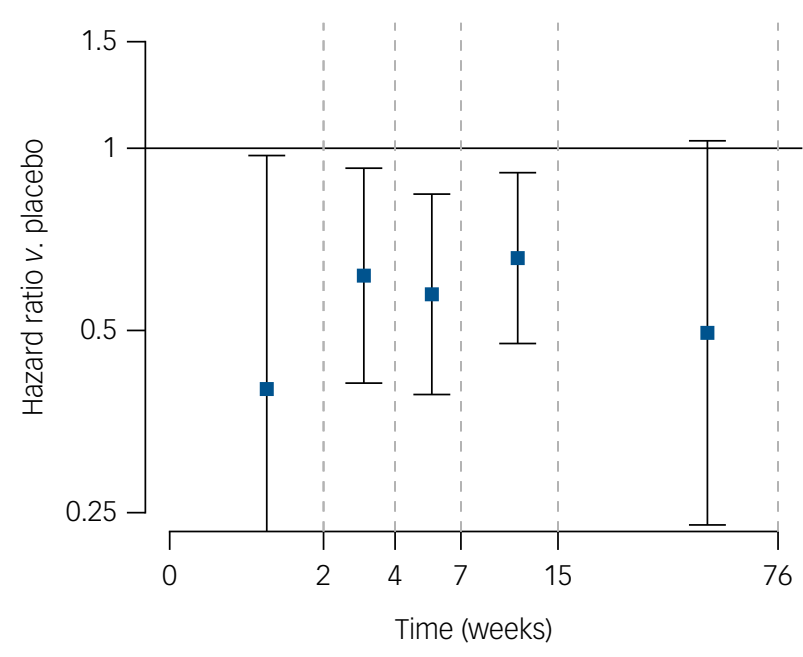

(c)

Manic episode

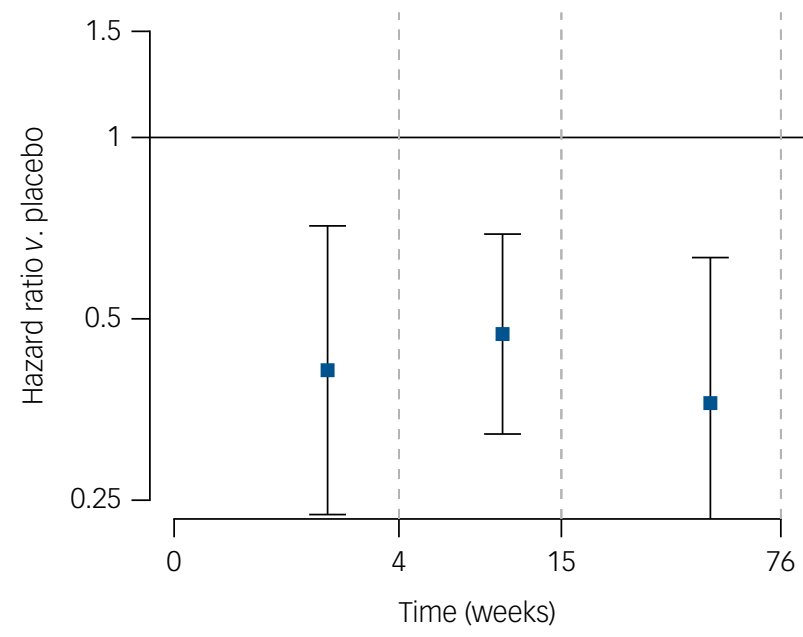

(b)

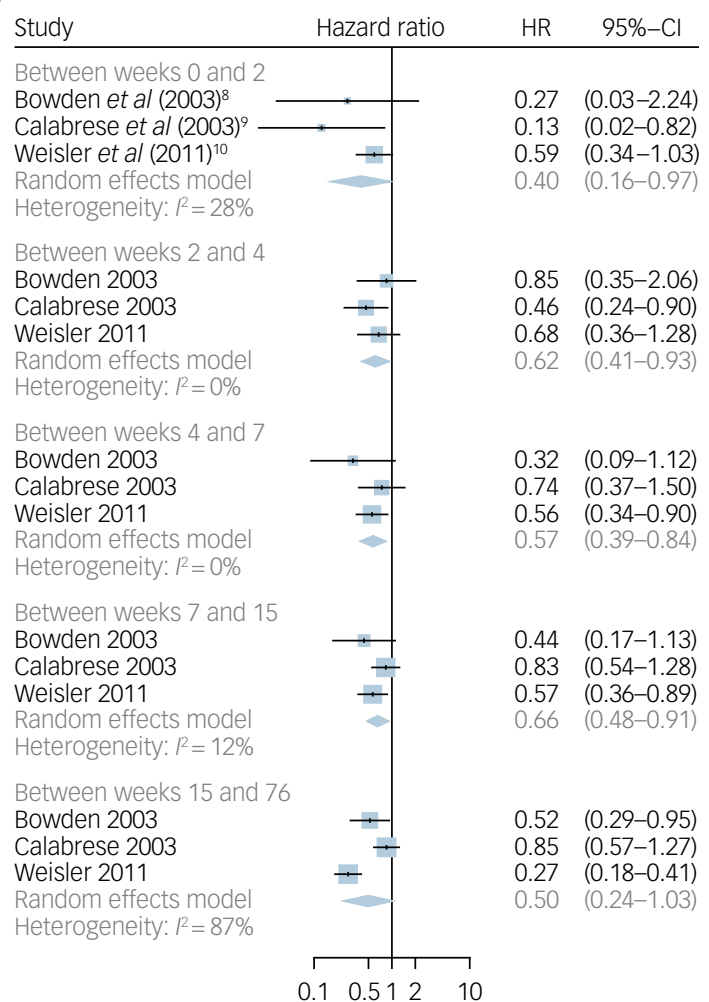

(d)

Depressive episode

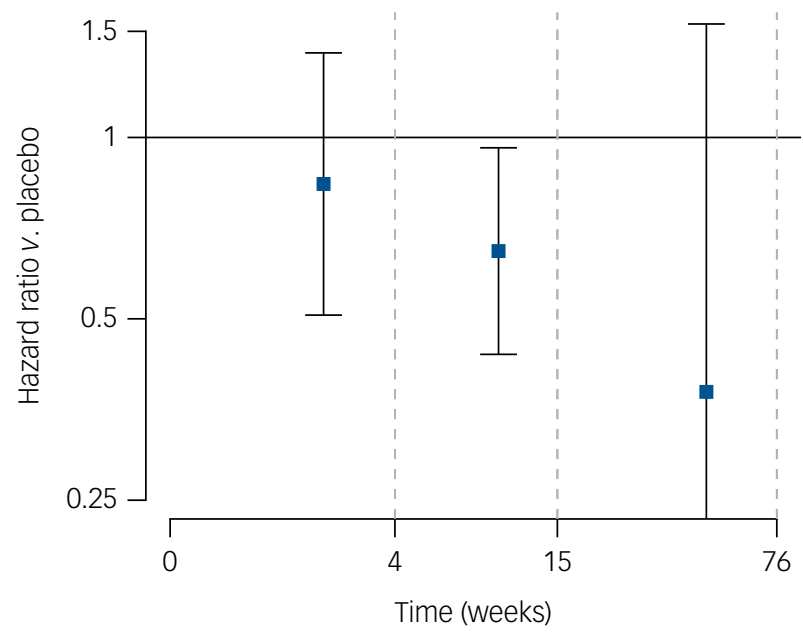

\section{Fig. 1 Effect of lithium on risk of relapse.}

(a) Effect of lithium versus placebo on risk of relapse in bipolar I disorder over time (weeks). Hazard ratios (HRs) with $95 \%$ Cls shown for relapse into any mood episode. Vertical dashed lines mark the boundaries of time intervals over which HR estimates were made. Horizontal axis logarithmic for clarity. (b) Forest plot of HR estimates over consecutive time intervals. (c) Effect on risk of relapse into manic episodes. (d) Effect on risk of relapse into depressive episodes.

$=0.47$ (95\% CI 0.32-0.69) and between week 15 and week $76(\mathrm{HR}=$ 0.36, 95\% CI 0.21-0.63) (Fig. 1(c)).

\section{Depressive relapse}

For depressive relapse, there was no significant reduction in relapse rates in those randomised to lithium between baseline and week 4 (HR $=0.84$, 95\% CI 0.51-1.38), however, rates of depressive relapse were lower in the lithium group between weeks 4 and 15 $(\mathrm{HR}=0.65,95 \%$ CI $0.44-0.96)$ but not statistically significant between week 15 and week $76(\mathrm{HR}=0.38,95 \%$ CI $0.09-1.54)$ (Fig. 1(d)). Heterogeneity estimates were lower for manic relapse
( $I^{2}$ between 0 and $\left.42 \%\right)$ than for depressive relapse ( $I^{2}$ between 32 and $86 \%)$

\section{Discussion}

This analysis combined data from three placebo-controlled randomised clinical trials of lithium for relapse prevention in bipolar disorder. People with bipolar I disorder randomised to lithium rather than placebo have a lower risk of relapse evident within the first 2 weeks of treatment. Considering poles of illness separately, the full effect to reduce manic relapse appears evident within the first 
4 weeks ( $\mathrm{HR}=0.41,95 \%$ CI $0.24-0.73)$, however, early effects on depressive relapse are not clearly demonstrated $(\mathrm{HR}=0.84,95 \%$ CI 0.51-1.38).

The magnitude of the early preventative effects found here are similar to the overall relative risks (RRs) previously described ${ }^{1}$ for both any relapse $(\mathrm{RR}=0.66,95 \% \mathrm{CI} 0.53-0.82)$ and manic relapse specifically $(\mathrm{RR}=0.52,95 \% \mathrm{CI} 0.38-0.71)$. These data indicate that in future studies of the mechanism of action of lithium in preventing relapse in bipolar disorder, antimanic mechanisms should be apparent within 4 weeks of use, however, the mechanisms underlying its effects against depressive relapse may take longer to become apparent.

The temporal resolution of this analysis is restricted by the size of underlying studies, and the risk of relapse over time, and the underlying review was not designed specifically to identify timing of onset data. If there were a substantial delay in onset of relapse prevention effects within the first 2 weeks, one would expect the overall estimate for that period to be lower than for later intervals. This does not on initial inspection seem to be the case for total relapse or manic relapse specifically. However, the results here are consistent with a slower onset of preventative effects for depressive relapse. These results avoid any bias by enrichment design, as trial participants were not preselected for acute episode response to lithium. However, the conventional approach in reporting relapse in trials in bipolar disorder may tend to underestimate longer-term benefits against depressive relapse because of differential withdrawal from study follow-up, ${ }^{11}$ and limited numbers of depressive relapses recorded lead to reduced statistical power.

It has been suggested that beneficial preventative effects of lithium may take years to become fully apparent. ${ }^{3}$ Very late-onset effects may not be well captured by typical clinical trials given their duration, so such effects may need to be investigated by other methods, through the use of large observational data-sets such as those becoming available through the use of electronic health records. ${ }^{12}$

These data indicate that there is an early onset of lithium relapse prevention effects in bipolar disorder, particularly against manic relapse. These early effects are similar in magnitude to longerterm estimates previously demonstrated. Full effects against depressive relapse may develop over a longer period.

\footnotetext{
Matthew J. Taylor, MRCPsych (iD), Consultant Psychiatrist, University Department of Psychiatry, Warneford Hospital, Oxford OX3 7JX, UK and Clinical Senior Lecturer in Bipolar Disorder, Institute of Psychiatry, Psychology \& Neuroscience, King's College London, UK. Email: matthew.taylor@psych.ox.ac.uk

First received 21 May 2018, final revision 2 Aug 2018, accepted 4 Aug 2018
}

\section{Supplementary material}

Supplementary material is available online at https://doi.org/10.1192/bjp.2018.177.

\section{References}

1 Severus E, Taylor MJ, Sauer C, Pfennig A, Ritter P, Bauer M, et al. Lithium for prevention of mood episodes in bipolar disorders: systematic review and metaanalysis. Int J Bipolar Disord 2014; 2: 15.

2 Alda M. Lithium in the treatment of bipolar disorder: pharmacology and pharmacogenetics. Mol Psychiatry 2015; 20: 661-70.

3 Ahrens B, Müller-Oerlinghausen B, Grof P. Length of lithium treatment needed to eliminate the high mortality of affective disorders. Br J Psychiatry 1993; 163 (suppl 21): 27-9.

4 Lenox RH, Wang L. Molecular basis of lithium action: integration of lithiumresponsive signaling and gene expression networks. Mol Psychiatry 2003; 8: 135-44.

5 Taylor MJ, Freemantle N, Geddes JR, Bhagwagar Z. Early onset of selective serotonin reuptake inhibitor antidepressant action: systematic review and meta-analysis. Arch Gen Psychiatry 2006; 63: 1217-23.

6 Parmar MKB, Torri V, Stewart L. Extracting summary statistics to perform metaanalyses of the published literature for survival endpoints. Stat Med 1998; 17: 2815-34.

7 Tierney JF, Stewart LA, Ghersi D, Burdett S, Sydes MR. Practical methods for incorporating summary time-to-event data into meta-analysis. Trials 2007; 8: 16.

8 Bowden C, Calabrese J, Sachs G, Yatham L, Ashgar S, Hompland M, et al. A placebo-controlled 18-month trial of lamotrigine and lithium maintenance treatment in recently manic or hypomanic patients with bipolar I disorder. Arch Gen Psychiatry 2003; 60: 392-400.

9 Calabrese J, Bowden C, Sachs G, Yatham L, Behnke K, Mehtonen O, et al. A placebo-controlled 18-month trial of lamotrigine and lithium maintenance treatment in recently depressed patients with bipolar I disorder. J Clin Psych 2003; 64: 1013-24.

10 Weisler RH, Nolen WA, Neijber A, Hellqvist A, Paulsson B. Trial 144 Study Investigators. Continuation of quetiapine versus switching to placebo or lithium for maintenance treatment of bipolar I disorder (Trial 144: A randomized controlled study). J Clin Psych 2011; 72: 1452-64.

11 Licht RW, Severus E. Letter to the editor: is it valid to break down results from long-term trials in bipolar disorder by polarity of relapses? Int J Bipolar Disord 2014; 2: 8.

12 Hayes JF, Marston L, Walters K, Geddes JR, King M, Osborn DP. Lithium vs. valproate vs. olanzapine vs. quetiapine as maintenance monotherapy for bipolar disorder: a population-based UK cohort study using electronic health records. World Psychiatry 2016; 15: 53-8. 